Courrier du Centre international Blaise Pascal

$7 \mid 1985$

Varia

\title{
Papiers de famille
}

\section{Francine Leclercq}

\section{OpenEdition \\ Journals}

Édition électronique

URL : http://journals.openedition.org/ccibp/435

DOI : $10.4000 /$ ccibp.435

ISSN : 2493-7460

\section{Éditeur}

Centre international Blaise Pascal

\section{Édition imprimée}

Date de publication : 5 avril 1985

Pagination : 13-14

ISSN : 0249-6674

\section{Référence électronique}

Francine Leclercq, " Papiers de famille », Courrier du Centre international Blaise Pascal [En ligne],

7 | 1985, mis en ligne le 27 novembre 2015, consulté le 03 mai 2019. URL : http://

journals.openedition.org/ccibp/435 ; DOI : 10.4000/ccibp.435

Ce document a été généré automatiquement le 3 mai 2019.

Centre international Blaise Pascal 


\title{
Papiers de famille
}

\author{
Francine Leclercq
}

\section{Deux codicilles inédits de Marguerite Périer}

1 Bien que leur texte n'ait pas encore été publié, le contenu de ces deux codicilles était néanmoins connu car Élie Jaloustre, dans son étude sur Marguerite Périer ${ }^{1}$, les avait longuement évoqués. Il en avait eu connaissance grâce aux Archives hospitalières de Clermont qu'il avait dépouillées et qui permettent d'éclaircir certains passages assez obscurs, en particulier sur la destination des legs de 800 livres et 2000 livres que Pierre de Grandsaigne devra utiliser "ainsy que ladite demoiselle Périer luy a déclaré ses intentions ». Par cette formule sibylline, Marguerite Périer rappelle que ces sommes devront être remises à un certain Dieudonné qui s'était plaint auprès d'elle d'avoir été jadis frustré de son héritage par le chanoine Louis Périer, frère de la testatrice au cas où les prétentions de celui-ci s'avéreraient justifiées².

2 Sans doute n'est-il pas inutile de préciser que Françoise Rochenoux, dont il est question dans le codicille du 19 février, occupait un logement dans la maison de Cournon que Marguerite Périer avait vendue à l'Hôpital général de Clermont. Par son testament rédigé en 1720, Marguerite avait déjà demandé que celle-ci puisse, sa vie durant, conserver la jouissance de ce logement.

3 Par l'expression « son héritier » la testatrice désigne « les pauvres de l'Hôpital général de cette ville de Clermont » qu'elle a institués " ses héritiers universels ${ }^{3}$ ", en les distinguant d'avec les nombreux légataires qu'elle a énumérés: couvents, pauvres des paroisses, domestiques, débiteurs de sa famille, exceptant nommément ses cousins germains d'Issoire, Messieurs Aubert, qu'elle estimait avoir été trop bien servis ${ }^{4}$. La distribution de l'héritage s'annonçait assez épineuse. C'est pourquoi dans le testament de 1720 Marguerite Périer «supplie» son exécuteur Monsieur Fournier «d'avoir la bonté de prévenir les contestations qui pourraient arriver entre [ses] légataires et [ses] héritiers ». M. Fournier ne figure pas dans les codicilles de 1733 mais on reconnaît la même prudence juridique et la même détermination passionnée dans la manière dont elle garantit l'indépendance de Pierre de Grandsaigne en l'investissant de sa pleine confiance. 
4 L'orthographe de l'original a été respectée, seule la ponctuation a été modernisée afin de rendre le texte plus intelligible.

\section{Codicille du 19 février 1733 (A.D. Puy-de-Dôme, 5E 38 DEP 501)}

Legs par Marguerite Périer à Françoise Rochenoux, de Cournon, d'une pension annuelle de 2 septiers de blé et à Gabrielle Rochenoux, sa sœur, de Clermont, d'une pension de 25 livres, pensions qui ne seront versées qu'après la mort de la testatrice et qui s'éteindront au décès de la première des deux sœurs, legs également à Pierre de Grandsaigne, seigneur des Champs, de 800 livres qu'il devra utiliser selon les instructions qu'il en a reçues.

Pardevant le notaire royal en la ville de Clermont-Ferrand en Auvergne soussigné et en présence des témoins cy après nommés, mandez expressement à l'effet des présentes, fut présente demoiselle Marguerite Périer, fille majeure demeurante en cette ville de Clermont, parroisse du Port, laquelle se voyant au lict malade de maladie corporelle, seine de ses sens, mémoire et entendement, ainsy qu'il est aparu audit notaire et auxdits témoings à ce mandez expressement, laquelle a dit et déclaré avoir cy devant fait son testament olographe, lequel a esté expistographé par devant Meynial et qui est au pouvoir de Sauvat, notaire en cette ville, qui en est saisy, de la datte tant dudit testament que dudit espitographe elle n'est pas mémorative, mais déclare se ressouvenir de la teneur de sondit testament, et lequel voulant codiciler, comme elle codicile par ces présentes, en conséquence a le présent codicile volontairement fait; et par iceluy elle donne et lègue à Françoize Rochenoux, fille majeure demeurante de présent à Cornon, une pension viagère de deux septiers bled consoigle et Gabrielle Rochenoux, sa sœur, demeurante en cette ville, une pension viagère de la somme de vingt cinq livres, et lesquelles pensions prendront cours du jour du décès de ladite demoiselle Périer et par son héritier institué par sondit testament leurs seront payées annuellement jusques au décès de la première mourante desdites Françoize et Gabrielle Rochenoux, et par ce décès de la première mourante, ladite demoiselle Périer pour raisons à elle connues veut et entend que lesdites deux pensions viagères de deux septiers bled, d'une part, et de vingt cinq livres, d'autre, par elle léguées par le présent codicile demeurent pleinement et entièrement esteintes et amortyes sans que la survivante desdites Françoize et Gabrielle Rochenoux puissent après le décès de la prémourante demander la continuation de la pension viagère à elles cy dessus léguée. De plus ladite demoiselle Périer veut et ordonne par le présent codicile que par son héritier institué par sondit testament il soit délivré dans l'année de son décès la somme de huit cent livres à $\mathrm{M}^{\text {re }}$ Pierre de Grandsaigne, écuyer, sieur Deschamps, demeurant en cette ville, paroisse du Port, pour par luy employer ladite somme de huit cent livres ainsy que ladite demoiselle Périer luy a déclaré ses intentions et lesquelles elle est très convaincue que ledit Sieur Deschamps exécutera en leurs entier et sans que ledit sieur Deschamps soit tenu de les déclarer ny en rendre aucun compte à qui que ce soit, pour quelque cause et occasion que ce puisse estre, et dont elle l'a déchargé et décharge par ces présentes. Et au surplus ladite demoiselle Périer a confirmé la teneur de sondit testament, déclare que ces présentes est son codicile qui luy a esté lu et relu par ledit notaire en présence desdits temoings cy assemblez, mandés et venus à la réquizition de ladite demoiselle Périer, seule laquelle l'a trouvé conforme à sa volonté et veut qu'il soit exécuté suivant sa forme et teneur. Et à l'exécution d'iceluy elle oblige, affecte et hypotèque tous les biens de sa succession, renonçant à tout ce qui pourroit estre à ce contraire; a prié et requis lesdits temoings de vouloir s'en ressouvenir. Car ainsy, etc. Fait et passé audit Clermont dans la maison de ladite demoiselle Périer et au devant de son lict l'an mil sept cent trente trois et le dix neufième de février, après midy, en présence de $\mathrm{M}^{\mathrm{e}}$ Antoine Grimaud, marchand, de $\mathrm{M}^{\mathrm{e}} \mathrm{Jean}$ Tamen, marchand confiturier, de sr Jean Laporte, maître perruquier, et sr Antoine Michel et aussy maître perruquier et de sr 
Charles Dufour, garçon perruquier originaire de la ville d'Aurillac, demeurant et travaillant ches ledits sr Laporte, tous témoins demeurans en cette ville de Clermont qui ont signé avec ladite demoiselle Périer.

[au bas sont les signatures] GRIMAUD M. PERIER J. TAMEN

LAPORTE MICHELET DUFOUR

CHAUDESSOLLE notaire royal

\section{Codicille du 27 février 1733 (A.D. Puy-de-Dôme, 5E 38 DEP 501)}

Legs à Pierre de Grandsaigne d'une somme de 2000 livres qu'il devra utiliser selon les instructions qu'il a reçues.

Pardevant le notaire royal en la ville de Clermont-Ferrand en Auvergne soussigné et en présence des témoings cy après nommés mandez expressément à l'effet des présentes, fut présente demoizelle Margueritte Périer, fille majeure demeurante en cette dite ville de Clermont, parroisse du Port, laquelle se voyant au lict malade de maladie corporelle, seine de ses sens, mémoire et entendement ainsy qu'il est apparu audit notaire et auxdits témoings à ce mandez expressément, laquelle a dit et déclaré avoir cy devant fait son testament olographe, lequel épistographé pardevant Meynial, notaire, et est à présent au pouvoir de Sauvat, notaire en cette ville, de la datte duquel testament et épistographe elle n'est pas mémorative, mais déclare se ressouvenir de la teneur de sondit testament. Comme aussy s'étant faite représenter par le notaire soussigné son codicile par elle fait le dix neuf du présent mois de février, par lequel il est porté entre autres choses qu'elle veut et entend qu'il soit délivré par son héritier, dans l'an de son décès, à $\mathrm{M}^{\text {re }}$ Pierre de Grandsaigne, écuyer, sr Des Champs, demeurant en cette ville, une somme de huit cent livres pour l'employer par ledit sieur Deschamps suivant les intentions qu'elle luy a déclaré, sans que ledit sieur Deschamps soit tenu d'en rendre aucun compte, dont elle l'a déchargé ; ladite demoiselle Périer a derechef par ces présentes codicile [s], tant sondit testament que son dit codicile du dix neuf du présent, et par le présent codicile elle veut et ordonne que dans la même année de son décès il soit délivré par sondit héritier, outre et pardessus la susdite somme de huit cent livres portée par son précédent codicile, une autre somme de deux mille livres audit sieur de Grandsaigne, sieur Deschamps, pour icelle employer de même suivant les intentions que ladite demoiselle Périer luy a déclarées, estant de même convaincue qu'il voudra bien prendre cette peine et sans que ledit sieur de Grandsaigne soit tenu de faire aucune déclaration desdites intentions ny qu'il soit tenu d'en rendre aucun compte à qui que ce soit, pour quelque cause et occasion que ce puisse estre, dont ladite demoiselle Périer l'a déchargé et décharge en tant que besoin est ou seroit ; veut aussy et ordonne ladite demoiselle Périer que outre et au par dessus les susdites deux sommes cy dessus ordonnées estre délivrées audit sieur de Grandsaigne aux fins que dessus; elle veut encore que son héritier soit obligé d'exécuter toutes les autres intentions que ledit sieur de Grandsaigne luy déclarera de sa part. Et au surplus elle veut et entend que sondit testament et sondit précédent codicile sortent leurs plein et entier effet, déclare que ces présentes est son codicile qui luy a esté lu et relu par ledit notaire, en présence desdits témoings cy assemblez, mandés et venus à la réquizition de ladite demoiselle Périer, laquelle l'a trouvé conforme à sa volonté et veut qu'il soit exécuté suivant sa forme et teneur et à l'exécution d'iceluy elle oblige, affecte et hypothèque tous les biens de sa succession, renonçant à tout ce qui pourrait estre à ce contraire; a prié et requis lesdits témoings de vouloir s'en ressouvenir. Car ainsy l'a voulu ladite demoiselle Périer, fait et passé audit Clermont dans sa maison et au devant de son lict l'an mil sept cent trente trois et le vingt sept de février après midy en présence de $\mathrm{M}^{\mathrm{e}}$ Antoine Grimaud, marchand, de $\mathrm{M}^{\mathrm{e}}$ Jean Tamen, marchand confiturier, de sr Jean Laporte, maître perruquier, sr Antoine Michelet, aussy maître perruquier et de sieur Charles Dufour, garçon perruquier originaire de la ville d'Aurillac, demeurant 
et travaillant ches ledit sieur Laporte, tous témoings demeurans en cette dite ville de Clermont qui ont signé avec ladite demoiselle Périer.

[au bas sont les signatures] GRIMAUD J. TAMEN M. PERIER

LAPORTE MICHELET DUFOUR

CHAUDESSOLLE notaire royal

\section{NOTES}

1. Élie JALOUSTRE, Une nièce de Pascal : Marguerite Périer, dans Bulletin historique et scientifique de l'Auvergne, 1901, p. 68-96, 102-147. Le testament daté du 4 décembre 1720 est donné p. 139-147 avec deux codicilles datés l'un du 4 décembre 1720, l'autre du 2 mai 1721, d'après la copie conservée aux Archives hospitalières. L'autographe en quatre pages se trouve en fait aux Archives départementales, minutes du notaire Meyniel, Sauvat successeur, Cote 5E/ 11 DEP 1044.

2. Voir Jaloustre, op. cit., p. 136-139.

3. Dans Jaloustre, op. cit., p. 145.

4. Ibid., p. 144-145.

\section{INDEX}

Mots-clés : Perrier (Marguerite), codicille 